\title{
Bewährung der Gleichheit. Dialektik und radikale Demokratietheorie
}

\author{
Andreas Gelhard
}

Hegel's criticism of Kant's philosophy of representation can be understood as a shift from representation (Vorstellung) to presentation (Darstellung). This shift affects not only theoretical but also practical philosophy. A basic idea of Hegel's Phenomenology is that freedom can only be realized if individuals present themselves as free. Hegel often calls this conflictual process of presentation "probation" or "verification" (Bewährung). In acts of practical verification, individuals do not simply assert their identity, but carry out a liberating break with given identities. This motive was very important for Adorno's philosophy of the non-identical, but was lost in the following generations of the Frankfurt School. The essay shows that it currently lives on in Jacques Rancière's theory of radical democracy. It argues that Rancière is closer to some important intuitions of early Critical Theory than Axel Honneth's theory of recognition. To this end, it examines a discussion between Honneth and Rancière that took place in Frankfurt in 2009.

Bewährung; Dialectic; Disidentification; Democracy; Hegel; Honneth; Equality; Freedom; Rancière; Recognition

Andreas Gelhard became professor of philosophy of education at Friedrich Wilhelms Universität Bonn in October 2018, after four years as an assistant professor of philosophy and educational theory at the department of philosophy at the University of Vienna. Before taking up the positions in Vienna and Bonn, he was scientific director of the Forum for Interdisciplinary Research (TU Darmstadt) and science editor at Suhrkamp Verlag (Frankfurt am Main).

Recent publications: Skeptische Bildung. Prüfungsprozesse als philosophisches Problem, Zürich, Berlin: diaphanes 2018. Kritik der Kompetenz, third revised edition, Zürich, Berlin: diaphanes 2018. Epistemische Tugenden. Zur Geschichte und Gegenwart eines Konzepts, Tübingen: Mohr [ed. with Ruben Hackler and Sandro Zanetti; forthcoming].

\section{Einführung}

Jamais l'égalité n'existerait que dans sa vérification. Jacques Rancière

Die Bezüge zwischen den verschiedenen Generationen der Frankfurter Schule sind locker. Das zeigt sich nirgends deutlicher als im Bedeutungsverlust des Begriffs Dialektik. Was bei Adorno noch die Grundlinie des gesamten philosophi- 
schen Programms vorgab, spielte schon bei Habermas keine Rolle mehr. Das eröffnet Spielräume für eine neue Besetzung des Begriffs Dialektik. Dabei sind natürlich sehr unterschiedliche Strategien denkbar. Ich möchte im Folgenden nur einen möglichen Ansatzpunkt diskutieren, der auf den ersten Blick vielleicht überraschen wird. Im Jahr 2009 fand in Frankfurt am Main eine kurze Debatte zwischen Axel Honneth und Jacques Rancière statt. Zur Vorbereitung des Gesprächs dienten je ein kritischer Essay, in dem sich Honneth und Rancière mit der Position ihres Gesprächspartners auseinandersetzen. ${ }^{1}$ Darin zeigt sich Rancière nicht nur sehr offen für das Vokabular der Anerkennung, sondern entwickelt auch eine eigene Deutung der „Dialektik der Anerkennung“.2 Man kann das für eine bloße rhetorische Finte halten. Im Verlauf des Gesprächs zeigen sich aber so deutliche Berührungspunkte zwischen Rancières politischer Theorie und einer negativistisch gewendeten - Dialektik, dass es sich lohnt, die Sache etwas näher zu betrachten.

Rancière hat wichtige Grundzüge seiner politischen Theorie in Auseinandersetzung mit einem Autor der Aufklärung entwickelt. Seine zentrale These, dass man Gleichheit nicht als Ziel eines Lernprozesses, nicht als Produkt von Erziehung und auch nicht als Resultat irgendeiner anderen Form des „Erwerbs“ betrachten kann, formuliert er erstmals ausführlich in seiner Darstellung der AntiPädagogik Joseph Jacotots, die konsequent von der Gleichheit der Intelligenzen ausgeht. Vieles spricht dafür, dass Rancière in dieser Studie erstmals den Kern seiner politischen Theorie entwickelt. ${ }^{3}$ Nach Rancière formuliert Jacotot eine Kritik an der pädagogischen Ordnung des Erklärens, deren Bedeutung weit über den Bereich des so genannten Bildungssystems hinausreicht. Die Ungleichheit zwischen Lehrer und Schüler erscheint aus seiner Perspektive als Paradigma einer Strategie, die es erlaubt, Ungleichheit als „Rückstand“ aufzufassen, den es erst einmal aufzuholen gilt. ${ }^{4}$ Wer dieser Dynamik des endlosen Aufschubs von Gleichheit entgehen will, der darf, so Rancières zentrale Einsicht, Gleichheit

1 Das Gespräch und die begleitenden Texte sind dokumentiert in: Katia Genel / JeanPhilippe Deranty (Hg.), Recognition or Disagreement. A critical Encounter on the Politics of Freedom, Equality, and Identity, New York 2016. Alle Übersetzungen der Zitate aus diesem Band sind meine eigenen.

2 Jacques Rancière, „Critical Questions on the Theory of Recognition“, in: Genel / Deranty (Hg.), Recognition or Disagreement, S. 83-95, hier: S. 90. Zu Rancières Gebrauch des Anerkennungsbegriffs in seinen früheren Schriften vgl. Jean-Philippe Deranty, „Between Honneth and Rancière: Problems and Potentials of a Contemporary Critical Theory of Society“, in: ebd., S. 33-80, hier: S. 36 f.

3 Vgl. Jacques Rancière, Der unwissende Lehrmeister. Fünf Lektionen über die intellektuelle Emanzipation, 2. überarbeitete Auflage, Wien 2009.

4 Ebd., S. 140. In dieser Ordnung der Ungleichheit wartet man vergeblich darauf, die Gleichheit durch Lernen zu erwerben. Emblem für den endlosen Aufschub der Gleichheit ist der Satz „Ich erkläre es dir nächstes Jahr“ (ebd., S. 33). 
Andreas Gelhard

nicht als Produkt politischer oder pädagogischer Lernprozesse setzen. Gleichheit kann nicht als ein „Ziel“ von Emanzipation verstanden werden, das bei hinreichender Bemühung irgendwann erreicht wird, sondern nur als „unbedingt anzunehmende Voraussetzung aller Emanzipation“. 5

Die These von der unbedingten Voraussetzung der Gleichheit ist unvereinbar mit einer postmetaphysischen Teleologie, die sozialen Konflikten ,eine Art von praktisch-moralischem Lernpotential“ zuschreibt. ${ }^{6}$ Sie ist Teil einer radikalen Demokratietheorie, die die Idee einer „konsensuellen Demokratie“ als Widerspruch in sich betrachtet. ${ }^{7}$ Nach Rancière verwirklicht sich Demokratie in Akten der Emanzipation, in denen sich die Anteilslosen einer bestimmten sozialen Ordnung ihren Anteil aneignen. Solche Akte sind nur möglich, wenn es gelingt, den Streit zwischen der bestehenden Ordnung und einer alternativen Ordnung, in denen die Anteile anders verteilt sind, öffentlich zu führen. Honneths Idee sozialer Lernprozesse ist aus dieser Perspektive doppelt problematisch. Sie droht nicht nur den Tag, an dem die Subjekte alle Kompetenzen erworben haben, die sie zur Teilhabe am politischen Prozess befähigen, endlos aufzuschieben; sie tendiert auch dazu, Konflikte in „Probleme“ zu verwandeln, die nicht im politischen Kampf ausgefochten, sondern mit Hilfe von „Expertenwissen“ gelöst werden. ${ }^{8}$

Die Auflösung von politischen Konflikten in Problemlösungsprozesse ist nach Rancière nicht mit dem Begriff der Demokratie vereinbar. Seine Theorie der Demokratie setzt Gleichheit nicht als Ziel, sondern als unbedingte Voraussetzung politischen Handelns. Sie bleibt aber nicht bei der bloßen Beschwörung dieser unbedingten Voraussetzung stehen. Rancières historische Untersuchungen zur Geschichte der Arbeiterbewegung ziehen vielmehr die Konsequenz aus der Einsicht, dass die Voraussetzung der Gleichheit in konkreten Akten der Aneignung von Gleichheit bewährt werden muss. Als zweites entscheidendes Moment neben der supposition der Gleichheit nennt er ihre vérification in Akten, die den politischen Dissens inszenieren. Diese Akte der vérification müssen als dramatische Akte verstanden werden, in denen Subjekte mit den ihnen zugewiesenen Identitäten brechen. In Das Unvernehmen, das man als Rancières erstes Hauptwerk ansehen kann, spricht er daher von einer vérification de l'égalité. ${ }^{9}$

5 Tobias Klass, „Depotenzierungen eines Nicht-Wissenden: Lektionen über Emanzipation“, in: Alfred Schäfer (Hg.), Hegemonie und autorisierende Verführung, Paderborn 2014, S. 113-136, hier: S. 133.

6 Vgl. Axel Honneth, Kampf um Anerkennung Zur moralischen Grammatik sozialer Konflikte, Frankfurt/M. 1994, S. 42.

7 Vgl. Jacques Rancière, Das Unvernehmen. Politik und Philosophie, Frankfurt/M. 2002, S. 105.

8 Vgl. ebd., S. 119.

9 Rancière, Das Unvernehmen, S. 51; Rancière, La Mésentente. Politique et Philosophie, Paris 1995, S. 64. 
Bewährung der Gleichheit. Dialektik und radikale Demokratietheorie

Ich möchte hier vorschlagen, Rancières Begriff der vérification, der üblicherweise etwas formalistisch als „Verifizierung“ wiedergegeben wird, mit Bewährung zu übersetzen. Dieser Vorschlag geht davon aus, dass eine enge Korrespondenz zwischen Rancières Gedanken einer vérification de l'égalité und Hegels Theorie einer Befreiung zur Gleichheit besteht. In der Phänomenologie des Geistes formuliert Hegel eine dialektische Darstellung der Beziehung zwischen der unbedingten Voraussetzung von Freiheit und ihrer praktischen Bewährung, die deutliche Korrespondenzen zu dem Zusammenspiel von supposition und vérification in Rancières politischer Theorie zeigt. Ich werde im Folgenden einige Grundzüge dieser Konstellation herausarbeiten. Sie hat, wie ich glaube, einige Aufmerksamkeit verdient, weil sie es erlaubt, die negativistischen - im eigentlichen Sinne dialektischen - Züge von Hegels praktischer Philosophie hervortreten zu lassen, ohne dabei auf mehr oder weniger moderate teleologische Muster zurückgreifen zu müssen.

\section{Der Begriff der Bewährung (Vorstellen vs. Darstellen)}

Der Begriff der Bewährung gehört zu den zentralen philosophischen Konzepten, durch die Hegel den Primat der Vorstellung in Kants kritischer Philosophie bricht. ${ }^{10}$ In Kants Kritik der reinen Vernunft stehen alle zentralen Begriffe - von Empfindung und Anschauung bis zu Begriff und Idee - unter der gemeinsamen Gattung der „Vorstellung überhaupt (repraesentatio)“ (B $376 \mathrm{f}$.). Das entspricht Kants erkenntnistheoretischer Orientierung an der newtonschen Physik. Wenn Kant die „Welt“ in seiner transzendentalen Dialektik als „Inbegriff aller Erscheinungen“ (B 447) bestimmt, so bestimmt er sie als das Ganze aller möglichen Gegenstände der Erfahrung. Unter „Erfahrung“ kann dann folglich nur noch die Erkenntnis von Gegenständen verstanden werden. Der Vollzugscharakter des Erfahrungsbegriffs, der ihm nicht nur in der aristotelischen Tradition zukam, sondern der auch zu Kants Zeit für den alltäglichen Gebrauch des Ausdrucks

10 Folgt man Béatrice Longuenesse, so kann man Hegels Philosophie als eine einzige groß angelegte Kritik am Repräsentationsdenken Kants begreifen: vgl. Hegel et la critique de la métaphysique, deuxième édition revue et augmenté, Paris 2015, S. 30-33. Werke Kants werden - mit römischer Band- und arabischer Seitenzahl - nach der Akademieausgabe zitiert: Akademieausgabe. Kants gesammelte Schriften, herausgegeben von der Berlin-Brandenburgischen Akademie der Wissenschaften, Berlin $1902 \mathrm{ff}$. (abweichend nur Zitate aus der Kritik der reinen Vernunft, wie üblich mit A und B nach der ersten und zweiten Auflage). Werke Hegels werden - mit der Sigle TW, römischer Band- und arabischer Seitenzahl - nach der Theorie Werkausgabe des Suhrkamp Verlages zitiert: G.W.F. Hegel, Werke, auf der Grundlage der Werke von 1832-1845 neu edierte Ausgabe, Redaktion: Eva Moldenhauer und Karl Markus Michel, Frankfurt/M. 1986 (abweichend nur Zitate aus der Phänomenologie des Geistes mit der Sigle PG nach der Ausgabe des Meiner Verlages, Hamburg 1988). 
Andreas Gelhard

„Erfahrung“ kennzeichnend war, fällt in dieser transzendentalphilosophischen Bestimmung aus. Erfahrung ist, mit Kants kürzester Formel gesprochen, ,empirische Erkenntnis“.

Hegels Revision von Kants Erfahrungsbegriff in der Phänomenologie des Geistes gibt der Erfahrung den Vollzugscharakter zurück, der in ihrer Bestimmung als empirische Erkenntnis verloren gegangen war. Auffallendstes Kennzeichen dieser Revision ist die gesteigerte Aufmerksamkeit für die Bedeutung sprachlicher Vollzüge in epistemischen und praktischen Kontexten. Schon im ersten Kapitel der Phänomenologie hängt alles davon ab, dass wir das „Dieses“, durch das wir auf einen Gegenstand verweisen, nicht „vorstellen“, sondern „aussprechen“ (vgl. PG 71). Die ganze Tragweite dieser Verschiebung vom Vorstellen zum Sprechen wird aber erst klar, wenn man berücksichtigt, wie Hegel das Grundprinzip seines dialektischen Denkens - die Isosthenie, das Gleichgewicht der Gründe - aus einer Analyse sprachlicher Vollzüge gewinnt. Die sprachliche Äußerung fungiert in Hegels Dialektik als Paradigma einer viel weiterreichenden Dynamik der Entäußerung, durch die wir uns unausweichlich den Reaktionen, den Antworten und dem Widerstand anderer aussetzen. Eigentliches Thema des ersten Kapitels der Phänomenologie ist daher die Eigenart der Sprache, die in ihr ausgesprochene Meinung „unmittelbar zu verkehren, zu etwas anderem zu machen" (PG 78).

Die Figur der Verkehrung gleichgewichtiger Sätze und Mächte markiert in Hegels Theorie der Erfahrung den Moment der Aporie, aus dem sich das Selbst nicht zu befreien vermag, indem es einzelne Ansichten über einen Gegenstand oder einzelne Erwartungen an eine Handlungssituation korrigiert. Das Gleichgewicht der Sätze und Mächte verlangt vielmehr eine grundsätzliche Revision der Maßstäbe des Erkennens und Handelns, die sich als untauglich zur Auflösung des Gegensatzes erwiesen. Erfahrung gewinnt dadurch wieder den Charakter einer freien Selbstprüfung, den die Leserinnen und Leser Hegels immer wieder mit dem Ausdruck der Bewährung bezeichnet haben. ${ }^{11}$

Die berühmteste Stelle der Phänomenologie des Geistes, an der Hegel den Begriff der Bewährung gebraucht, ist der Abschnitt über Selbständigkeit und Unselbständigkeit des Selbstbewussteins, der Hegels Überlegungen zur Dialektik der Anerkennung eröffnet. Hegel zeigt dort, dass sich Freiheit nur verwirklichen kann, indem sich ein Selbst als frei darstellt. An diesem Punkt des Argumentationsganges kann das nur geschehen, indem die Beteiligten ihre absolute Unabhängigkeit von jeder Bindung - selbst von der Bindung ans eigene Leben - demonstrieren und sich im Kampf auf Leben und Tod „bewähren“ (PG 130). Diese

11 Vgl. Andreas Gelhard, Skeptische Bildung. Prüfungsprozesse als philosophisches Problem, Zürich / Berlin 2018, Kap. IV. Für die neuere Hegel-Literatur: Dina Emundts, Erfahren und Erkennen: Hegels Theorie der Wirklichkeit, Frankfurt/M. 2012; Für die ältere Literatur: Martin Heidegger, Hegels Phänomenologie des Geistes. Freiburger Vorlesung Wintersemester 1930/31, 3. Aufl., Frankfurt/M. 1997. 
Analyse der Selbstbehauptung im Kampf bleibt nicht Hegels letztes Wort zum Thema der Anerkennung. Die Bewährung der Freiheit im Kampf eröffnet nur eine weit gespannte Darstellung von praktischen Konflikten, die erst am Ende des Geist-Kapitels - in einem Verhältnis der Anerkennung unter Gleichen - zu einem ersten Abschluss findet. ${ }^{12}$ Die Darstellung von Erfahrung als Prozess der Bewährung bleibt in all diesen Analysen aber durchgehend leitend. Dabei geht Hegel durchgehend davon aus, dass die philosophische Darstellung von Erkenntnis- und Handlungsvollzügen nur darin besteht, dem Sichdarstellen des Selbst in praktischen Prozessen eine artikulierte Form zu geben. Die Phänomenologie des Geistes versucht die philosophische Artikulation einer Welt, die nicht mehr als Korrelat von Erkenntnis, sondern als Ort des Handelns begriffen wird. Diese Welt ist nicht mehr nur ein Ganzes von Erscheinungen, die mit Kant als mögliche Gegenstände der Erkenntnis zu begreifen wären, sondern zugleich der Ort des handelnden Erscheinens - der Entäußerung -, in der sich das Selbst immer wieder dem Blick, dem Urteil und dem Widerstand anderer aussetzt.

Auch Kant kennt den Begriff einer bewohnten Welt, in der praktische Ansprüche miteinander in Konflikt geraten können. In seiner Anthropologie bestimmt er Welt als „Dasein eines Ganzen anderer, mit mir in Gemeinschaft stehender Wesen“ (AA VII 130). Vor allem Martin Heidegger hat auf die Diskrepanz zwischen diesem anthropologischen Weltbegriff und dem metaphysischen Weltbegriff der transzendentalen Dialektik aufmerksam gemacht. In einer seiner Freiburger Vorlesungen bemerkt er, Kant greife zwei Weltbegriffe aus der philosophischen Tradition auf, lasse sie aber unvermittelt „nebeneinander stehen“. ${ }^{13}$ Während die transzendentale Dialektik Welt als Horizont von Gegenstandserkenntnis fasst, formuliert die Anthropologie eine Form von „Weltkenntnis“, für die „Welt“ in erster Linie „Miteinandersein“ bedeutet. ${ }^{14}$ Weltkenntnis haben nicht transzendentale Subjekte, sondern die Bewohner eines geteilten Ganzen, die sich zu diesem Ganzen als „Weltbürger“ verhalten. ${ }^{15}$ Will man diese Diagnose terminologisch fixieren, so kann man mit Pirmin Stekeler-Weithofer sagen, dass es Kant nicht gelingt, die Beziehung zwischen der „Bezugswelt“ empirischer Erkenntnis und der „Vollzugswelt“ freien Handelns konsistent zu bestimmen. ${ }^{16}$

12 Ich komme in Abschnitt 4 darauf zurück.

13 Martin Heidegger, Einleitung in die Philosophie. Freiburger Vorlesung Wintersemester 1928/29, Frankfurt/M. 1996, 248.

14 Ebd., 298, 300.

15 Der Pluralism der Denkungsart, den Kant in seiner Anthropologie den verschiedenen Spielarten des Egoism gegenüberstellt, bezeichnet die „Denkungsart“: ,sich nicht als die ganze Welt in seinem Selbst befassend, sondern als ein bloßer Weltbürger zu betrachten und zu verhalten“ (AA VII 130).

16 Vgl. Pirmin Stekeler-Weithofer, Philosophie des Selbstbewußtseins. Hegels System als Formanalyse von Wissen und Autonomie, Frankfurt/M. 2005, S. 150. 
Andreas Gelhard

Vieles in Hegels dialektischem Denken kann aus dem Versuch verstanden werden, genau diese Beziehung zwischen Bezugswelt und Vollzugswelt philosophisch darzustellen. Mit der abstrakten Trennung zwischen metaphysischem und anthropologischem Weltbegriff fällt dabei auch die Grenze zwischen dem theoretischen und dem praktischen Teil der kritischen Philosophie. Das eröffnet die Möglichkeit einer praktischen Reinterpretation zentraler Begriff von Kants Erkenntnistheorie. Besonders deutlich zeigt sich das im Falle des Begriffs der Erscheinung, den Hegel in praktischen Bezügen als Bewährung bezeichnet. Kant, folgt in seiner Reflexion über Freiheit einer klaren Dichotomie zwischen Phänomenalem und Intelligiblem, die es erlaubt, die „Kausalität aus Freiheit“ dem Einzugsbereich der Naturkausalität zu entziehen. Das hat zur Folge, dass Freiheit im Rahmen von Kants transzendentalphilosophischer Konstruktion nicht erscheinen kann. Dem setzt Hegel eine Theorie der Freiheit entgegen, die mit jeder Form von Kausalitätsdenken bricht. Hegel denkt Freiheit als ein Erscheinen unter anderen, das sich gegen deren Identitätszumutungen als selbstständig bewähren muss. Zur vorläufigen Erläuterung dieses Gedankens sei hier nur eine Passage der Heidelberger Enzyklopädie zitiert, die das Erscheinen im Handeln als Geschehen der Bewährung fasst:

„Nicht bloß in Beziehung auf Gott, sondern auch in sonstiger Beziehung geschieht es häufig, daß man sich der Kategorie des Wesens in abstrakter Weise bedient und dann bei Betrachtung der Dinge das Wesen derselben als ein gegen den bestimmten Inhalt ihrer Erscheinung Gleichgültiges und für sich Bestehendes fixiert. Man pflegt so namentlich zu sagen, es komme bei den Menschen nur auf ihr Wesen an und nicht auf ihr Tun und ihr Betragen. Darin liegt nun zwar das Richtige, daß dasjenige, was ein Mensch tut, nicht in seiner Unmittelbarkeit, sondern nur als vermittelt durch sein Inneres und als Manifestation seines Innern zu betrachten ist. Nur darf dabei nicht übersehen werden, daß das Wesen und dann weiter das Innere sich eben nur dadurch als solche bewähren, daß sie in die Erscheinung heraustreten“ (TW VIII 234).

Dieser Begriff der Bewährung markiert das Zentrum von Hegels Kritik an Kants Repräsentationsdenken. ${ }^{17}$ „Bewährung“ im Sinne Hegels bezeichnet kein Geschehen der Vorstellung, sondern eines der Darstellung. Besonders deutlich hebt das Jean-Luc Nancy in einer seiner Hegel-Studien hervor. Nancy verwendet den von Hegel immer wieder gebrauchten Begriff der Manifestation, um den spezifischen Modus eines Erscheinens zu bestimmen, das nicht die Setzung von Gegenständen, sondern ein Sich-Aussetzen an andere bezeichnet. ${ }^{18}$ Entsprechend kontrastiert er das kantische Denken der Vorstellung, das auf die eine oder andere Weise immer ein Subjekt in Beziehung zu „seinem“ Objekt setzt, mit einem

$17 \mathrm{Zu}$ den handlungstheoretischen Implikationen der zitierten Passage vgl. Robert B. Pippin, Hegel's Practical Philosophy, Cambridge / New York 2008, S. 150-157.

18 Vgl. Jean-Luc Nancy, Hegel. L'inquiétude du négatif, Paris 1997, S. 47-51. 
Denken der Darstellung, das den dramatischen Aspekt des Erscheinens unter anderen hervorhebt. ${ }^{19}$

\section{Die Dramatik des Dissenses}

Hegel eröffnet das sechste Kapitel der Phänomenologie mit dem für jede Lektüre des Buches zentralen Hinweis, dass die in den vorangehenden Kapiteln dargestellten Gestalten des Bewusstseins als bloße „Abstraktionen“ des konkreten sozialen Zusammenhangs zu betrachten sind, den Hegel als „Geist“ bezeichnet (PG 289). Entsprechend kann das Geist-Kapitel nicht einfach die Darstellung von Bewusstseinsgestalten fortsetzen; sein Gegenstand sind nun nicht mehr Bewusstseinsgestalten, sondern „Gestalten einer Welt“ (PG 290). Damit exponiert Hegel einen Begriff von Welt, der sich weder auf den Weltbegriff der transzendentalen Dialektik noch auf den der Anthropologie reduzieren lässt. Er bestimmt die Welt als bewohnte Welt, die sich durch die Pluralität von Lebensformen in konfligierende „Welten“ teilt (ebd.). Er erschließt so eine Sphäre sozialer Antagonismen, die Kant als anthropologische von den antinomischen Strukturen des metaphysischen Weltbegriffs trennt. Nach Hegel ist die Welt der Antagonismen keine andere als die, die sich in kosmologischer Perspektive als antinomische begreifen lässt. Wenn Adorno die philosophische Radikalität von Hegels Dialektik in der methodischen Direktive sieht, dass sich Vermittlung nicht ,zwischen“ entgegengesetzten Extremen, sondern „in“ den Extremen selbst vollzieht, so markiert er damit sehr präzise den Punkt, an dem Hegel den transzendentalphilosophischen Grundriss von Kants Antinomik der reinen Vernunft sprengt. ${ }^{20}$ Kants Vorstellung von vier kosmologischen Problemen, die sich nur im Widerstreit von These und Antithese formulieren lassen, liefert die entscheidende Einsicht, dass Weltbegriffe notwendig widersprüchlich werden. Erst die Erkenntnis, dass Weltbegriffe von Bewohnern einer Welt gebildet werden, denen es um die Orientierung in der Welt geht, lässt aber „das dialektische Moment“ in Hegels Sinn des Wortes zu Tage treten: Wenn Bewohner einer Welt das Ganze

19 „Le savoir sera donc, non pas une représentation (Vorstellung: position d'objet devant un sujet-de-savoir et pour lui, conforme à sa ,vision des choses', c'est-à-dire à sa pauvre limitation), mais une présentation (Darstellung: ,position-là', mise en place et en scène, exposition, surgissement de l'être-sujet en tant que tel), et par conséquent la négation de toute présence donnée, qu'elle soit d',objet' ou de ,sujet'““ (ebd., S. 18).

20 ,Vermittlung heißt [...] bei Hegel niemals, wie das verhängnisvolle Mißverständnis seit Kierkegaard es sich ausmalt, ein Mittleres zwischen den Extremen, sondern die Vermittlung ereignet sich durch die Extreme hindurch in ihnen selber; das ist der radikale, mit allem Moderantismus unvereinbare Aspekt Hegels" (Theodor W. Adorno, Drei Studien zu Hegel, in: Gesammelte Schriften, Bd. 5, Frankfurt/M. 2003, S. 247-381, hier: S. 257). 
Andreas Gelhard

bestimmen, in dem sie leben, geraten sie notwendig in Konflikt mit anderen Bestimmungen dieses Ganzen, die mit demselben Anspruch auf Ausschließlichkeit auftreten; wenn sie das Ganze bestimmen, in dem sie leben, finden sie den Widerspruch ,in allen Gegenständen aller Gattungen, in allen Vorstellungen, Begriffen und Ideen“" (TW VIII 128).

Dialektik im Sinne Hegels besteht nicht darin, diese Gegenstände, Vorstellungen und Begriffe in einem großen Ganzen aufgehen zu lassen; sie besteht in dem systematischen Nachweis, dass die begriffliche Bestimmung des großen Ganzen - der Welt - unweigerlich konkurrierende Bestimmungen desselben Ganzen produziert, die sich nicht widerstandslos miteinander versöhnen lassen. Die spezifisch moderne Strategie, mit diesem Problem umzugehen, nennt Hegel „Bildung“. Prozesse der Bildung reagieren auf die spezifisch moderne Erfahrung, dass „Welt“, sobald man sie bestimmt, ,zerfällt“ (PG 323).

Man muss sich an dieser Stelle vor Augen führen, wie weit der Weltbegriff der Phänomenologie des Geistes von Kants kosmologischen Überlegungen entfernt ist. Hegel denkt Welt niemals nur als Totalitätsbegriff, der das Denken vor besondere Herausforderungen stellt, sondern immer auch als den Ort des Denkens. Der Mensch bildet Weltbegriffe als Weltbürger. Dadurch legt er immer auch eine Vorstellung von dem Ganzen fest, in das er sich mit anderen teilt. Das betont auch Heidegger in seiner Auseinandersetzung mit Kants Weltbegriff. ${ }^{21}$ Da er Kants anthropologischen Weltbegriff gegen den kosmologischen Weltbegriff mobilisiert, verliert er aber die Dimension des Konflikts aus dem Blick, die für Hegels dialektisches Denken entscheidend ist. Sucht man nach einem zeitgenössischen Pendant des Weltbegriffs, den Hegel unter dem Titel der Bildung entwickelt, so entspricht er am ehesten Rancières Konzept einer „Aufteilung des Sinnlichen““. ${ }^{22}$

Rancière nutzt den doppelten Sinn des Wortes teilen (partager), um zwei Aspekte der bewohnten Welt zu unterscheiden, die Hegel unter den Titeln der Sittlichkeit und der Bildung behandelt: Eine Aufteilung des Sinnlichen erzeugt zugleich die Kontinuität eines „,geteilten Gemeinsamen“ und diskrete „Teile“ dieses Gemeinsamen, die als „Anteile“ nicht jedem zukommen. ${ }^{23}$ „Sinnlich“ ist diese Aufteilung, weil sie Welt als einen Wahrnehmungsraum erzeugt: Sie entscheidet darüber, was als Gegenstand wahrgenommen, als Stimme vernommen und als zugehörig gezählt wird. ${ }^{24}$ Natürlich stellt Rancière die Herstellung dieser Aufteilung nicht dialektisch dar. Aber er betont die Zweideutigkeit der Teilhabe, die diese Aufteilung des Sinnlichen nur durch Ausschluss anderer Aufteilungen

21 Vgl. z. B. den $\S 14$ der Einleitung in die Philosophie (,Wir teilen uns in die Unverborgenheit des Seienden").

22 Vgl. Jacques Rancière, Die Aufteilung des Sinnlichen, 2. Aufl., Berlin 2008.

23 Vgl. ebd., S. 12.

24 „Diese Verteilung der Anteile und Orte beruht auf einer Aufteilung der Räume, Zeiten und Tätigkeiten, die die Art und Weise bestimmen, wie ein Gemeinsames sich der Teilhabe öffnet und wie die einen und die anderen daran teilhaben“ (ebd., S. 12 f.). 
behaupten kann: „Eine ,gemeinsame“ Welt ist niemals bloß ethos im Sinne eines gemeinsamen Aufenthaltsortes, der sich aus der Sedimentierung einer bestimmten Anzahl verflochtener Handlungen ergibt. Sie ist immer auch eine konfliktreiche Verteilung von Seinsweisen und „Beschäftigungen“ in einem Möglichkeitsraum.“25

In der Phänomenologie des Geistes markiert diese Einsicht den Übergang von der Sittlichkeit zur Bildung. Unter dem Titel der Sittlichkeit analysiert Hegel eine Welt, deren Bewohner unmittelbar wissen, welcher Lebensform sie angehören und welchem Gesetz des Zusammenlebens sie folglich unterworfen sind. Das sittliche Bewußtsein entscheidet sich nicht für eine Lebensform, es „ist entschieden“, so schreibt Hegel, einer Welt „anzugehören“ (PG 305). Hegels berühmte Interpretation der Antigone zeigt, dass diese unmittelbare Entschiedenheit für eine Lebensform nicht bloße Gefangenschaft unter einem auferlegten Gesetz bedeutet. Antigones Auflehnung gegen Kreon lässt ein Moment der Autonomie in der Sphäre der Sittlichkeit aufbrechen, indem sie ihr Gesetz gegen seines - das Gesetz der Familie gegen das des Staates - ins Feld führt. Diese Einsicht bricht in Hegels Darstellung vor allem dort durch, wo er die Sprache des Bildungsabschnitts verwendet, um die Konflikte in der Sphäre der Sittlichkeit zu beschreiben. Nur aus der Warte einer Welt, die die spezifisch moderne Dynamik der Entzweiung und Verkehrung kennt, hat es Sinn, das Verhalten des sittlichen Bewusstseins, das noch keine inneren Konflikte kennt, als „unentzweites Verhalten zum Gesetze“ zu bezeichnen (PG 307). In dieser Perspektive lässt sich bereits $a n$ den antiken Figuren eine Dynamik ablesen, die erst unter dem Titel der Bildung ins Bewusstsein der Akteure übergeht: Antigones Ansprüche lassen sich nur in der Verletzung fremder Ansprüche - das heißt: als Schuld - verwirklichen. Das „unentzweite“ Verhalten zum eigenen Gesetz kann sich nur als „Entzweiung“ mit dem Gesetz des Anderen realisieren, es kann nur ,dem einen Gesetze zugewandt" handeln, sofern es dem anderen ,abgesagt“ hat und es durch sein Handeln „verletzt“ (PG 308).

Nach Christoph Menke besteht die eigentlich philosophische Bedeutung des Geständnisses, durch das Antigone die Ordnung der Sittlichkeit „stört“, in der Demonstration, dass das Spiel der Mächte, aus dem die bestehende Herrschaft hervorgegangen ist, nur scheinbar zur Ruhe gekommen ist:

„Kult und Tragödie sind Medien eines Gedächtnisses des Unterlegenen. Dadurch werden sie zu Instanzen der Revision des geschichtlichen Prozesses. Nicht jedoch, indem sie den Sieg der neuen, politischen, über die alten, naturhaften Götter durch ein anderes Urteil ersetzen, sondern indem sie, was historisch ebenso einseitig wie eindeutig entschieden

25 Ebd., S. 66. 
Andreas Gelhard

war, in die Situation eines unbeendbaren Konfligierens gleicher Mächte zurückversetzen. Die Tragödie nimmt einen Kampf wieder auf, der längste beendet erschien“. ${ }^{26}$

Die Tragödie lässt das Gleichgewicht der Mächte, aus dem eine herrschende Ordnung hervorgegangen ist, in dieser Ordnung wieder aufbrechen. Darin liegt ihr Vorgriff auf eine Moderne, in der der Versuch, den Konflikt der Mächte endgültig zu beenden, immer wieder durch die antinomische Verfassung der Welterkenntnis und den antagonistischen Charakter des Sozialen eingeholt worden ist. Wie in Hobbes' Modell des Gesellschaftszustandes die Gewalt des Naturzustandes zurückkehrt - der Souverän behält als einziger sein „Recht auf alles“ - so kehrt der Streit metaphysischer Positionen, den Kant durch die Kritik der reinen Vernunft in einen ewigen Frieden überführen will, in dieser Kritik als Antinomik wieder. ${ }^{27}$ Hegels Entgrenzung von Kants Antinomik zu einer konsequent dialektischen Philosophie kann vor diesem Hintergrund als erster Versuch verstanden werden, einen philosophischen Weltbegriff $\mathrm{zu}$ formulieren, der dem immer wieder aufbrechenden Gleichgewicht der Mächte gerecht wird. Der Konflikt zwischen diesen Mächten kann im Selbstbewusstseins-Kapitel der Phänomenologie noch als ein agonales Geschehen zwischen Individuen verstanden werden, das durch Sieg und Niederlage entschieden wird. Im Kapitel über den Geist sind dagegen von vornherein „Welten“ im Spiel, die je den Anspruch auf Alleinherrschaft erheben. Die Reaktivierung des Konflikts kann daher nur als Reaktivierung eines Antagonismus verstanden werden, der dieselbe Totalität unter entgegengesetzte Bestimmungen bringt. ${ }^{28}$ Begreift man diese Welten mit Rancière als Aufteilungen des Sinnlichen, so lässt sich dieser Antagonismus mit Rancières Konzept des politischen Streits erläutern: Dieser Streit inszeniert „die Konfrontation zweier Welten, die in einer einzigen beherbergt sind“; er trennt und verbindet „zwei Weisen des menschlichen Zusammenseins, zwei Typen der Aufteilung des Sinnlichen“. ${ }^{29}$

\section{Kampf um Anerkennung}

Die Bestimmung des Dissenses als Konflikt zwischen Welten markiert die Perspektive, aus der Rancière auch das Problem der Anerkennung in den Blick nimmt. Diese Perspektive unterscheidet sich signifikant von Honneths sozialpsychologischer Bestimmung des Anerkennungsbegriffs. Was den prinzipiellen

26 Christoph Menke, Tragödie im Sittlichen. Gerechtigkeit und Freiheit nach Hegel, Frankfurt/M. 1996, S. 121.

27 Vgl. Gelhard, Skeptische Bildung, Kap. III.

28 Ich übernehme die Unterscheidung zwischen Agonalem und Antagonismus von Oliver Marchart, Das unmögliche Objekt, Berlin 2013, Kap. II.

29 Rancière, Das Unvernehmen, S. 38 f. (Übers. modifiziert). 
Umgang mit dem Vokabular der Anerkennung angeht, so muss man zunächst festhalten, dass Rancière keine grundsätzlichen Vorbehalte gegen die Verwendung dieses Vokabulars hat. ${ }^{30}$ Seine Kritik gilt nicht dem Begriff der Anerkennung als solchem, sondern vor allem dem starken Begriff der Selbstidentität, der für Honneths Modell der Anerkennung - in jeder seiner Fassungen - kennzeichnend ist. Das normative Ideal, dem alle Analysen von Kampf um Anerkennung folgen, ist die „Möglichkeit einer ungestörten Selbstbeziehung“ “. ${ }^{31}$ Anerkennung erscheint in diesem Zusammenhang als ein intersubjektives Geschehen, in dem sich mehr oder weniger ungestörte Selbstverhältnisse ausbilden; im Falle des Gelingens „Selbstvertrauen“, ,Selbstachtung“ und „Selbstschätzung““. 32 Diese Orientierung an einem starken Ideal gelingender Selbstbeziehung ist für Honneth auch im Jahr 2009 noch verbindlich. ${ }^{33}$ Aus Rancières Sicht ist es vor allem deshalb problematisch, weil es politische Dynamiken auf moralische Selbstverhältnisse zurückführt und die Konflikte zwischen sozialen Ordnungen, die Rancière Dissens nennt, nur als Impulse in einem sozialen Lernprozess wahrnimmt. ${ }^{34}$

Honneths Modell der Anerkennung droht - so kann man Rancières Bedenken kurz zusammenfassen - das Moment des Konflikts aus dem „Kampf um Anerkennung“"zu verdrängen. Zur Konkretisierung dieses Arguments ist vor allem der Text interessant, den Rancière als Diskussionsgrundlage für das Gespräch mit Honneth verfasst hat. Wie, so fragt Rancière darin, muss der Kampf um Aner-

30 Er selbst gebraucht den Begriff der Anerkennung in Schriften wie La leçon d'Althusser und in La parole ouvrière: vgl. Deranty, „Between Honneth and Rancière“, S. 3639.

31 Honneth, Kampf um Anerkennung, S. 8.

32 Ebd., S. 211.

33 Rancière orientiert sich in seiner Auseinandersetzung mit Honneth vor allem an Kampf um Anerkennung. Man könnte daher vermuten, dass seine Kritik im Jahr 2009 nicht mehr den Kern von Honneths Konzept trifft. Robert Pippin hat gegen Honneths „sozialpsychologische“ Deutung von Anerkennungsverhältnissen in Kampf um Anerkennung auf die Bedeutung gesellschaftlicher Institutionen verwiesen (vgl. Pippin, Hegel's Practical Philosophy, S. 183 f., 202, 210). Die starke Betonung der institutionellen Ebene in Honneths letztem großen Buch kann als Reaktion auf diese Kritik gedeutet werden (vgl. Axel Honneth, Das Recht der Freiheit. Grundriss einer demokratischen Sittlichkeit, Berlin 2011). Rancière konnte dieses Buch zum Zeitpunkt des Frankfurter Gesprächs noch nicht kennen. Das ist aber nicht weiter von Bedeutung, weil das starke Konzept von Selbstidentität auch für Honneths spätere Auffassung von Anerkennung verbindlich bleibt. In der Diskussion mit Rancière hält er unmissverständlich fest: „In my opinion, we simply cannot do without the notion of an undistorted and complete selfrelationship“ (Axel Honneth / Jacques Rancière, „A Critical Discussion“, in: Genel / Deranty (Hg.), Recognition or Disagreement, S. 107-129, hier: S. 110.).

34 Vgl. Honneth, Kampf um Anerkennung, S. 42 f.; Honneth, Das Recht der Freiheit, S. 613-615. 
Andreas Gelhard

kennung verstanden werden, wenn die Vokabel des Kampfes mehr sein soll als eine entfernte Hegel-Referenz? Seine kritische Perspektivierung von Honneths Konzept leitet er dabei schon damit ein, dass er die Gemeinsamkeit der alltäglichen Verwendungsweisen des Anerkennungsbegriffs, von denen sich die philosophische Verwendung absetzen muss, in einer starken Vorstellung substantieller Identität situiert. ${ }^{35}$ Dem setzt er eine Bestimmung von Anerkennung entgegen, die auf die sozialen Bedingungen der Identifizierung fokussiert:

„Anerkennung [im alltäglichen Gebrauch des Wortes] ist ein Akt der Bestätigung. Im Gegensatz dazu konzentriert sich der philosophische Begriff der Anerkennung auf die Bedingungen, die hinter einer solchen Bestätigung stehen; er konzentriert sich auf die Konfiguration des Feldes, in dem Dinge, Personen, Situationen und Argumente identifiziert werden können. Er ist nicht die Bestätigung von etwas bereits Vorhandenem, sondern die Konstruktion der gemeinsamen Welt, in der Existenzen erscheinen und bestätigt werden. “36

Das Argument markiert einen entscheidenden Punkt, in dem Rancière Hegel näher ist als Honneth. Der Kampf um Anerkennung erscheint bei ihm nicht als ein „intersubjektives“ Geschehen, sondern als Konflikt zwischen Anerkennungsordnungen, die festlegen, als wer oder als was die Beteiligten unter diesen Bedingungen identifiziert werden können. Ich hatte oben schon bemerkt, dass sich der Weltbegriff, den Hegel im Geist-Kapitel der Phänomenologie entwickelt, als „Aufteilung des Sinnlichen“ im Sinne Rancières erläutern lässt. Das liegt vor allem daran, dass Rancière und Hegel die Welt gleichermaßen als einen Horizont des praktischen Erscheinens fassen: als Bühne, auf der nicht jeder als sozialer Akteur auftreten und sich als berechtigter Sprecher äußern kann. Weil das nicht jeder kann und weil also Alternativen denkbar sind, hat es in dieser Perspektive Sinn, von Welten zu sprechen, die miteinander in Konflikt geraten.

Ich glaube, es ließe sich zeigen, dass Hegel hier im Abstoß von Kants transzendentaler Dialektik genau die Ebene erschließt, die Rancière in seiner politischen Theorie eher unvermittelt ansteuert: Wenn Welt nicht als Horizont des Erkennens, sondern des Handelns begriffen werden soll, kann es nicht mehr um die antinomische Verfassung metaphysischer Weltbegriffe gehen, sondern darum, dass die Identifizierung von Personen, Situationen und Argumenten, die unter den Bedingungen einer bestimmten Welt erscheinen, umstritten sind. Wie ich oben schon gezeigt habe, liegt der Begriff des Streits, mit dem wir es dabei zu tun haben, nicht auf der Ebene einzelner Akteure, sondern auf der Ebene der Anerkennungsordnungen - der Welten -, die darüber entscheiden, was unter diesen Bedingungen als rationale Handlung, als artikulierte Äußerung etc. gilt. Darauf zielt Rancières Begriff des Dissenses: „Ein Dissens ist kein Interessen-, Mei-

35 Vgl. Rancière, „Critical Questions“, S. 84.

36 Vgl. ebd., S. 84 f. 
nungs- oder Wertekonflikt, sondern eine Teilung im ,Gemeinsinn“: ein Streit darüber, was gegeben ist, und über den Rahmen, in dem wir etwas als gegeben wahrnehmen." 37

Auf dieser Grundlage zeigt Rancière, dass man politische Subjektivierung nicht als Prozess der Identifizierung, sondern als Geschehen der dissensuellen Desidentifizierung denken muss. Diesen Grundgedanken kann man - in den konkreten Formulierungen, die Rancière ihm gibt - als negativistisch bezeichnen. Es scheint daher kein Zufall, wenn Rancière in der Zusammenfassung seines Vorschlags, wie der Kampf um Anerkennung zu denken sei, ausdrücklich von Dialektik spricht:

„Wir wissen, dass der Kampf um Anerkennung einfach als die Forderung eines bereits konstituierten Subjekts verstanden werden kann - und oft verstanden wurde -, in seiner Identität anerkannt zu werden. So gibt es z. B. eine Vorstellung von den Ansprüchen von Minderheiten als Ansprüchen auf Achtung ihrer Identität. Aber wir können diese Ansprüche auch - und ich denke, das ist das Herzstück der Dialektik der Anerkennung - als Ansprüche darauf begreifen, eine bestimmte Identität nicht zugewiesen zu bekommen. Ein Minderheitenanspruch ist nicht nur der Anspruch auf Anerkennung der eigenen Kultur und dergleichen, sondern auch ein Anspruch, gerade nicht als Minderheit betrachtet zu werden, die besonderen Regeln gehorcht und eine besondere Kultur hat. Es kann als Anspruch auf die gleichen Rechte und die gleiche Art von Respekt und Wertschätzung wie jeder andere angesehen werden, wie alle, denen keine besondere Identität zugewiesen wird. “" ${ }^{38}$

Im Zentrum von Rancières Bestimmung der Anerkennung steht nicht ein starker - und moralisch aufgeladener - Identitäts-Begriff, sondern ein Konzept politischer Subjektivierung, das auf den Bruch mit gegebenen Identitäten hinausläuft. Die Dissonanz zwischen Honneth und Rancière ist an dieser Stelle so deutlich, dass sie zur klärenden Konfrontation einlädt. Christoph Menke eröffnete das Gespräch zwischen Honneth und Rancière entsprechend mit der Frage, ob Honneth in seinem Anerkennungsmodell einen Ort für den Gedanken der dissensuellen Desidentifizierung sieht. ${ }^{39}$ Honneth gesteht solche Momente der Desidentifizierung auch in seinem Modell der Anerkennung zu, da der durch soziale Konflikte vorangetriebene Lernprozess immer wieder eine Überwindung eingefahrener Identitätsmuster verlangt. Diese Momente der Desidentifizierung sind für ihn aber ohne Einfluss auf den ,normativen Hintergrund“, vor dem die Konflikte als Beitrag zu einem sozialen Lernprozess erkennbar sind. Dieser normative Hintergrund bleibt für Honneth bestimmt durch das „Telos unver-

37 Jacques Rancière: „Wer ist das Subjekt der Menschenrechte?“, in: Christoph Menke / Francesca Raimondi (Hg.): Die Revolution der Menschenrechte. Grundlegende Texte zu einem neuen Begriff des Politischen, Berlin 2011, S. 474-490, hier: S. 483.

38 Rancière, „Critical Questions“, S. 90.

39 Vgl. Honneth / Rancière, „A Critical Discussion“, S. 108. 
Andreas Gelhard

zerrter Selbstverhältnisse“.${ }^{40}$ Honneth ist offenkundig schon zu weit entfernt vom negativistischen Denken Adornos, um dem Gedanken der dissensuellen Desidentifizierung mehr als eine marginale Rolle in Prozessen der Anerkennung zuzugestehen.

\section{Befreiung zur Gleichheit}

Christoph Menke hat in den Jahren nach dem Frankfurter Gespräch eine Reihe von Texten vorgelegt, die Hegels Philosophie der Freiheit als eine Theorie der Befreiung begreifen. ${ }^{41}$ Anders als andere Autorinnen und Autoren, die der dritten Generation der Frankfurter Schule zugerechnet werden, bleibt Menke dabei wichtigen Grundmotiven von Adornos Denken treu. Das schützt ihn vor einer weichen Teleologie à la Honneth, die sozialen Konflikten den Sinn zuschreibt, gesellschaftliche „Lernprozesse“ voranzutreiben. Menke mobilisiert zentrale Motive von Adornos negativer Dialektik, um Hegels Theorie der Freiheit als eine Theorie der Befreiung lesbar zu machen. Der programmatische Kern dieser Theorie besteht in der Einsicht, dass Freiheit als ein Prozess der Befreiung begriffen werden muss und sich der Prozesscharakter von Befreiung am besten mit Hegels Begriff der Bildung bestimmen lässt. ${ }^{42}$ Es zeigt sich, dass der Begriff der Bildung bei Hegel kein geradliniges Geschehen der Identitätsbildung bezeichnet, sondern eines, das die Positivität gesellschaftlicher Ordnungen - und also die durch diese Ordnungen festgelegten Identitäten - negiert. Der Begriff bezeichnet einen Prozess der Befreiung, den man als „Befreiung von der Identität“ begreifen kann. ${ }^{43}$ Dabei ist allerdings weit mehr im Spiel als die einfache Vorstellung eines individuellen Ausbruchs, der sich aus gesellschaftlichen Zwängen löst. Entscheidende Bedingung für die konkrete Bestimmung der Befreiung als Bildung ist die Einsicht, dass die sozialen Identitäten, von denen sich das Selbst befreit, Produkte von Prozessen der Individuation sind, die man ihrerseits als Prozesse der Befreiung begreifen muss: als Prozesse der Befreiung von Natur. Menke fasst das in die Formel, dass man die Bedingungen der Befreiung von sozialen Identitäten nur begreift, wenn man ihre ,Vorgeschichte“ berücksichtigt, die das Selbst aus der Abhängigkeit von der Natur befreit. ${ }^{44}$ Beide Dimensionen der Befreiung sind im

40 Vgl. ebd., S. $108 \mathrm{f}$.

41 Vgl. Christoph Menke, „Autonomie und Befreiung“, in: Thomas Khurana / Christoph Menke (Hg.), Paradoxien der Autonomie. Freiheit und Gesetz I, Berlin 2011, S. 149-184. Christoph Menke, „Hegels Theorie der Befreiung. Gesetz, Freiheit, Geschichte, Gesellschaft“, in: Gunnar Hindrichs / Axel Honneth (Hg.), Freiheit. Internationaler Hegel-Kongress 2011, Frankfurt/M. 2013, S. 301-320.

42 Menke, „Hegels Theorie der Befreiung“, S. 301-303.

43 Ebd., S. 309.

44 Ebd., S. 310. 
Spiel, wenn Hegel von Bildung spricht: Die Befreiung zur Identität des Selbst, die seine Unabhängigkeit von natürlichen Zwängen sichert, und die Befreiung von der Identität des Selbst, die positive soziale Ordnungen aufbricht. ${ }^{45}$

In der Phänomenologie des Geistes stellt Hegel dieses Geschehen der zweifachen Befreiung in einer zweifachen Analyse von Anerkennungsverhältnissen dar. Das Anerkennungsverhältnis zwischen Herr und Knecht erlaubt dem Herrn die Befreiung aus den Zwängen der Natur, indem er den Knecht für sich arbeiten lässt. Hegel erkennt in dieser Befreiung aber ein prekäres Geschehen, das die dauerhafte Befreiung aus Naturzwängen durch die Errichtung eines Systems sozialer Zwänge erkauft. Die Befreiung aus den Zwängen der Natur ist eine Befreiung zur Identität, die als gescheitert betrachtet werden muss, weil es sich um eine Befreiung zur Ungleichheit handelt. Der Kampf um Anerkennung kann nur als die unvermeidliche Vorgeschichte einer Befreiung zur Gleichheit betrachtet werden, die Hegel am Ende des Geistkapitels darstellt. ${ }^{46}$

Diese abschließende Analyse der Anerkennung konfiguriert das Verhältnis sozialer Gleichheit unter dem Schlagwort des Gewissens. Das kann die Lektüre auf die falsche Fährte führen, weil das Gewissen - bei Rousseau oder Kant - den Inbegriff der Innerlichkeit bedeutet. Man muss sich daher von vornherein vor Augen halten, dass das Gewissen bei Hegel ein politischer Begriff ist: Es bezeichnet die - ambivalente - Möglichkeit, sich im Namen individueller Freiheit von vorgegebenen institutionellen Ordnungen zu distanzieren. ${ }^{47}$ Diesem politischen Charakter des Gewissens entspricht auch Hegels Entscheidung, das Gewissen „als handelnd zu betrachten“ (PG 433). Produkt dieses Perspektivwechsels, der das Gewissen nicht als Inbegriff der Innerlichkeit, sondern als Entäußerung des Selbst fasst, ist ein Konflikt zwischen handelndem und urteilendem Bewusstsein, der die Analysen des Geist-Kapitels abschließt. Die Bewährung der Freiheit vollzieht sich in diesem Streit als Befreiung zur Gleichheit. Entscheidender Wendepunkt der gesamten Dialektik des Gewissens ist eine Erfahrung der Gleichheit des Gegenübers, die das handelnde Bewusstsein zu einem Schuldbekenntnis veranlasst. Laut Hegel gesteht das handelnde Bewusstsein dem urteilenden Bewusstsein nicht einfach seine Schuld, sondern ,spricht ihre Gleichheit von seiner Seite in seinem Geständnisse aus“ (PG 438).

45 Vgl. ebd.

46 Ich folge hier Menkes Rekonstruktion von Hegels Theorie der Befreiung: vgl. insbesondere „Autonomie und Befreiung“, S. 182-184. Menke entwickelt seine Überlegungen durchgehend in Bezug auf Hegels Spätwerk, nicht mit Blick auf die Phänomenologie. Sie sind aber ohne großen interpretatorischen Aufwand auf die entscheidende Achse zwischen dem vierten und dem sechsten Kapitel der Phänomenologie abbildbar, die die beiden Darstellungen des Kampfes um Anerkennung verbindet.

47 Das bemerkt auch Honneth, ohne den Gedanken aber in seine Konzeption von Anerkennungsverhältnissen aufzunehmen: vgl. Honneth, Das Recht der Freiheit, S. 109 f. 
Andreas Gelhard

Das klingt, als wäre die Herstellung eines Verhältnisses gegenseitiger Anerkennung nur eine Frage der richtigen Einsicht. Tatsächlich macht das sich bekennende Bewusstsein die Erfahrung der Gleichheit des Anderen aber - nur scheinbar paradox - dadurch, dass es die Einsicht in die Gleichheit der Beteiligten nicht teilt. Das handelnde Bewusstsein wagt sein Geständnis, weil es erkannt hat, dass ,die Sprache das Dasein des Geistes“ ist und es „erwartet“ folglich, „daß der Andere das seinige zu diesem Dasein beitrage" (PG 438). Das seinige zum Dasein des Geistes beizutragen bedeutet aber gerade nicht, die Erwartungen seines Gegenübers zu erfüllen. Denn nach Hegel besteht der Unterschied zwischen geistigen Beziehungen und natürlichen Kausalverhältnissen gerade darin, dass der Geist die Kapazität besitzt, den Einfluss des anderen „abzubrechen“ und sich in verwandelter Form anzueignen. ${ }^{48}$ Es ist daher kein bloßer dramatischer Schlenker, sondern ein systematisch höchst bedeutsamer Moment, wenn das Gegenüber des bekennenden Bewusstseins die erwartete Erwiderung verweigert. Erst durch diesen Bruch der Beziehung, der die ,reine Kontinuität des Allgemeinen“ mit der „absolute[n] Diskretion“ des Einzelnen konfrontiert, erweist sich die Beziehung der beiden tatsächlich als geistig (PG 441).

Ich kann hier nicht im Einzelnen darstellen, wie Hegel den Konflikt zwischen handelndem und urteilendem Bewusstsein darstellt, der nach der Verweigerung der Erwiderung schließlich in einer Szene des Verzeihens terminiert. Was Hegel in dieser Szene des Verzeihens auseinanderlegt, ist nichts anderes als die Grundstruktur des Geistes. Die Sprache ist das Dasein des Geistes und die Sprache des Gewissens ist nach Hegel durch genau die Dialektik von Handeln und Urteilen gekennzeichnet, die für geistige Verhältnisse charakteristisch ist. Hegel bestimmt diese Sprache als Mitte einander anerkennender Selbstbewusstseine, mutet uns aber zu, diese Mitte als eine Beziehung zu begreifen, die die Beteiligten aufrechterhalten, indem sie voneinander „ablassen“ (PG 442). Die verweigerte Erwiderung und das Widerfahrnis des Verzeihens sind zwei komplementäre Modi dieses „Ablassens“, das für Hegel so zentrale Bedeutung besitzt, dass er es in den abschließenden Ausführungen über das absolute Wissen wieder aufgreift (PG 522).

\section{Supposition, vérification}

Ich komme abschließend noch einmal auf die bekannte Stelle der Phänomenologie zurück, an der Hegels Bruch mit Kants Denken der Repräsentation im Begriff der Bewährung greifbar wird. Hegel bestimmt den Kampf um Anerkennung an dieser Stelle als ein Geschehen, in dem sich die Beteiligten als frei dar-

48 In der Wissenschaft der Logik schreibt er, es sei die „Natur des Geistes“, nicht äußere Ursachen „sich in ihn kontinuieren zu lassen, sondern sie abzubrechen und zu verwandeln" (TW VI 228). 
stellen oder als frei bewähren. Der Begriff der Bewährung kann dabei als eine Konkretisierung des Begriffs der Darstellung gefasst werden: Indem Hegel das Sich-Darstellen als Sich-Bewähren fasst, gibt er ihm den Charakter eines informellen Prüfungsgeschehens. Dieses Prüfungsgeschehen darf nun nicht - das ist für alles Weitere zentral - mit solchen Formen der Prüfung verwechselt werden, in denen sich jemand für etwas qualifiziert. ${ }^{49}$ Was im Kampf um Anerkennung auf die Probe gestellt wird, ist nicht das Individuum im Sinne eines einmaligen Charakters mit erzählenswerter Lebensgeschichte. Die Angabe von Charakterzügen und Erlebnissen ist insgesamt nicht das, was nach Hegel unsere Eigenart als freie Individuen ausmacht. Daher zielt die Bewährung im Kampf um Anerkennung ganz im Gegenteil darauf, unsere Unabhängigkeit von all diesen Eigenschaften unter Beweis zu stellen und - a limine - noch die stärkste Bindung-ansich zu lösen, die uns zu ,gegenständlichen“ Einzelwesen mit Eigenschaften macht: die Bindung ans „Dasein“ (PG 130).

Mit Blick auf die Dynamik der Bewährung am Ende des Geist-Kapitels heißt das, dass die Bewährung ,als frei“ in letzter Konsequenz die Grundstruktur einer - positiven - Bewährung ,als x“ zerstört. Hegel fasst das Verzeihen, der Etymologie des Wortes entsprechend, als „Verzichtleistung auf sich“ (PG 441). Der Akt des Verzeihens erscheint so als Vollzug einer praktischen Epoché, die den Anderen nicht nur ,als x“ - in diesem Falle: ,,als gut“ anerkennt -, sondern ihn, noch vor jeder möglichen Zuschreibung von Eigenschaften, frei lässt, indem sie auf ihr Urteil verzichtet. Das Verhältnis gegenseitiger Anerkennung stellt sich ein, indem ein Bewusstsein, das andere „als gut anerkennt“, „oder vielmehr“ - und diese Präzisierung ist entscheidend - sein „Urteil fahren läßt“ (ebd.). Schon der Kampf um Anerkennung im Abschnitt über Herrschaft und Knechtschaft zeigte, dass man den anderen zwar ,als Person“ mit bestimmten Eigenschaften anerkennen kann, dass seine Anerkennung ,als frei“ sich aber nicht mehr angemessen in einer

49 Mein Interesse an Hegels Begriff der Bewährung geht auf ein Projekt zurück, in dem ich versuche Prüfungsprozesse einer philosophischen Analyse zugänglich zu machen (vgl. Gelhard, Skeptische Bildung). In diesem Zusammenhang brauchte ich einen Begriff zur Bezeichnung von informellen Prüfungsprozessen, die nicht eigens als Prüfung veranstaltet werden, sondern im alltäglichen Handeln mitlaufen. Ich habe daher zu zeigen versucht, dass man mit Hegel schon die alltägliche Erfahrung als einen Prozess der Bewährung verstehen muss, dass man allerdings die spezifische Negativität dieser Prozesse der Bewährung hervorheben muss, wenn man sie von Regimes der systematischen Disziplinierung unterscheiden will, die den Prüfungscharakter des alltäglichen Handelns zur Kontrolle der Lebensführung nutzen (Max Webers einschlägige Analysen bringen diese Regimes mit dem calvinistischen Bewährungsgedanken in Verbindung). Ich habe deshalb vorgeschlagen, terminologisch zwischen negativer und positiver Bewährung zu unterscheiden (vgl. ebd., Kap. IV und V). Diese Unterscheidung kann ich im Rahmen dieses kurzen Aufsatzes nicht näher erläutern. Ich beschränke mich auf die Erläuterung der negativen Bewährung, die Hegels Gebrauch des Begriffs entspricht. 
Andreas Gelhard

„als“-Struktur ausdrücken lässt (vgl. PG 130 f.). Sich „als frei“ darzustellen bedeutete schon dort, seine Unabhängigkeit von jedem „,bestimmten Dasein“ - und das heißt: von jedem Erscheinen ,als x“ - zu demonstrieren. Dasselbe gilt nun $a$ fortiori für das Verhältnis der Verzichtleistung, in dem sich, nach der unaufhebbaren Ungleichheit der Dialektik von Herr und Knecht, nun erstmals ein Verhältnis gegenseitiger Anerkennung herstellt. Den Anderen ,als gut“ anzuerkennen, bedeutet gar nicht, ihn ,als etwas“ anzuerkennen, sondern vielmehr, das eigene Urteil - und mit ihm die gesamte ,als“-Struktur - fahren zu lassen. ${ }^{50}$

„Als wer“ oder „als was“ wir unter bestimmten Bedingungen anerkannt werden können, entscheidet nicht unser „intersubjektives“ Verhältnis zu einem Gegenüber, sondern die Ordnung der Anerkennung, die zu einem spezifischen Zeitpunkt unsere Welt ist. Für ein einzelnes Individuum hat das ,als“ keinen Sinn. Nur Individuen, die sich als Bewohner dieser Welt identifizieren, können in dieser Welt als $\mathrm{x}$ - als gut, kompetent und mit Rechten ausgestattet - anerkannt werden. Die als-Struktur fahren zu lassen heißt daher, mit einer Welt zu brechen. Es bedeutet, den Konflikt zu eröffnen, den Hegel als Zerrissenheit der modernen Welt und den Rancière als Dissens bezeichnet. Nach Rancière ist dieser Akt der dissensuellen Desidentifizierung immer wieder nötig, um die Gleichheit in der Ungleichheit aufbrechen zu lassen und die „Dialektik der Politik“ in Bewegung zu halten. ${ }^{51}$ Sich als gleich zu bewähren kann dabei nicht bedeuten, bestimmte Kompetenzen an den Tag zu legen, in denen sich eine besondere Befähigung zum demokratischen Handeln zeigt. Die Unmöglichkeit, diese Kompetenzen festzulegen und zu erheben macht für Rancière - im Gegenteil - das demokratische Prinzip selbst aus:

„Das ist es, was ich als demokratisches Prinzip bezeichnet habe: das Fehlen eines Kriteriums, das diejenigen, die dazu bestimmt sind, zu regieren, von denen unterscheidet, die dazu bestimmt sind, regiert zu werden. Wenn wir es umgekehrt lesen, dann bedeutet das die Voraussetzung einer Kompetenz derer, die keine spezifische Kompetenz haben, einer Kompetenz, die von allen geteilt wird. Das bedeutet für mich, dass das demokratische Prinzip nicht das Prinzip einer bestimmten Regierung ist. Es ist das Prinzip der Politik selbst." 52

Rancières Nähe zu Hegels Idee einer Befreiung zur Gleichheit markiert hier zugleich den Bruch mit dem wichtigsten hegelschen Motiv, das in Honneths Modell der Anerkennung überlebt hat: mit der Teleologie sozialer „Lernpro-

50 Alexander García Düttmann hebt das als „Alterität des Anerkennungsprozesses“ hervor, die „die Möglichkeit des Als, genauer: der Anerkennung als ... durchkreuzt" (Zwischen den Kulturen. Spannungen im Kampf um Anerkennung, Frankfurt/M. 1997, S. $122 \mathrm{f}$.).

51 Honneth / Rancière, „A Critical Discussion“, S. 113.

52 Ebd., S. 112 f. 
zesse“. Hegel mit Rancière zu lesen bedeutet, ihn konsequent negativistisch - und folglich nicht teleologisch - zu lesen. Kern dieser philosophischen Strategie ist es, die Geschichte der Erfahrung des Bewusstseins von dem pädagogischen Schema zu befreien, das dem dramatischen Geschehen des Dissenses die Gleichheit gegenseitiger Anerkennung als Ziel setzt. Diese tiefe Divergenz der beiden Theorien zeigt sich auch in Rancières Reserve gegen Honneths Rede von ,sozialen Pathologien“. 53 Nach Honneth kann man überall dort von sozialen Pathologien sprechen, wo die Mitglieder einer Gesellschaft „,verlernt“ haben, ,an maßgeblichen Formen der sozialen Kooperation teilzunehmen“. ${ }^{54}$ Dem liegt die Idee zugrunde, dass die freie Teilhabe an politischen Prozessen ein „Bündel elementarer Kompetenzen" verlangt, die gelernt, aber auch wieder verlernt werden können. ${ }^{55}$ Aus dieser Perspektive besteht eine Abhängigkeit demokratischer Prozesse von pädagogischen Maßnahmen, von der man nach Rancière gerade abstrahieren muss, wenn man Demokratie nicht zur Sache von Lehrern, Beratern und Experten machen will. Die Kompetenz derer, die keine spezifische Kompetenz haben, ist die Kompetenz, die unbedingte Voraussetzung von Freiheit zu bewähren. Sie realisiert sich nicht in Prozessen der positiven Bewährung, die vorgesteckte Anforderungen erfüllen, sondern in Prozessen der negativen Bewährung, die den Dissens zwischen Welten aufbrechen lassen. In dieser Perspektive bedeutet Demokratie nichts anderes als Bewährung der Gleichheit.

\section{Literaturverzeichnis}

Adorno, Theodor W.: Drei Studien zu Hegel, in: Gesammelte Schriften, Bd. 5, Frankfurt/ M. 2003.

Deranty, Jean-Philippe: „Between Honneth and Rancière: Problems and Potentials of a Contemporary Critical Theory of Society“, in: Deranty, Jean-Philippe / Genel, Katia (Hg.): Recognition or Disagreement. A critical Encounter on the Politics of Freedom, Equality, and Identity, New York 2016, 33-80.

Deranty, Jean-Philippe / Genel, Katia (Hg.): Recognition or Disagreement. A critical Encounter on the Politics of Freedom, Equality, and Identity, New York 2016.

Emundts, Dina: Erfahren und Erkennen: Hegels Theorie der Wirklichkeit, Frankfurt/M. 2012.

García Düttmann, Alexander: Zwischen den Kulturen. Spannungen im Kampf um Anerkennung, Frankfurt/M. 1997.

Gelhard, Andreas: Skeptische Bildung. Prüfungsprozesse als philosophisches Problem, Zürich, Berlin 2018.

Hegel, Georg Wilhelm Friedrich: Phänomenologie des Geistes, hrsg. von Heinrich Clairmont und Hans Friedrich Wessels, Hamburg 1988.

53 Vgl. ebd., S. 119.

54 Honneth, Das Recht der Freiheit, S. 157.

55 Ebd., S. 196. 
Andreas Gelhard

Hegel, Georg Wilhelm Friedrich: Werke, auf der Grundlage der Werke von 1832-1845 neu edierte Ausgabe, Redaktion: Eva Moldenhauer und Karl Markus Michel, Frankfurt/M. 1986.

Heidegger, Martin: Hegels Phänomenologie des Geistes. Freiburger Vorlesung Wintersemester 1930/31, 3. Aufl., Frankfurt/M. 1997.

Heidegger, Martin: Einleitung in die Philosophie. Freiburger Vorlesung Wintersemester 1928/29, Frankfurt/M. 1996.

Honneth, Axel, Das Recht der Freiheit. Grundriss einer demokratischen Sittlichkeit, Berlin 2011.

Honneth, Axel: Kampf um Anerkennung. Zur moralischen Grammatik sozialer Konflikte, Frankfurt/M. 1994.

Honneth, Axel / Rancière, Jacques: „A Critical Discussion“, in: Deranty, Jean-Philippe / Genel, Katia (Hg.): Recognition or Disagreement. A critical Encounter on the Politics of Freedom, Equality, and Identity, New York 2016, 107-129.

Kant, Immanuel, Kants gesammelte Schriften. Akademieausgabe, hrsg. von der BerlinBrandenburgischen Akademie der Wissenschaften, Berlin $1902 \mathrm{ff}$.

Klass, Tobias: „Depotenzierungen eines Nicht-Wissenden: Lektionen über Emanzipation“, in: Alfred Schäfer (Hg.), Hegemonie und autorisierende Verführung, Paderborn 2014, S. 113-136, hier: S. 133.

Longuenesse, Béatrice: Hegel et la critique de la métaphysique, deuxième édition revue et augmenté, Paris 2015.

Marchart, Oliver: Das unmögliche Objekt, Berlin 2013.

Menke, Christoph: Tragödie im Sittlichen. Gerechtigkeit und Freiheit nach Hegel, Frankfurt/M. 1996.

Menke, Christoph: „Autonomie und Befreiung“, in: Thomas Khurana / Christoph Menke (Hg.), Paradoxien der Autonomie. Freiheit und Gesetz I, Berlin 2011, S. 149184.

Menke, Christoph: „Hegels Theorie der Befreiung. Gesetz, Freiheit, Geschichte, Gesellschaft", in: Hindrichs, Gunnar / Honneth, Axel (Hg.), Freiheit. Internationaler Hegel-Kongress 2011, Frankfurt/M. 2013, S. 301-320.

Nancy, Jean-Luc: Hegel. L'inquiétude du négatif, Paris 1997.

Pippin, Robert B.: Hegel's Practical Philosophy, Cambridge, New York 2008.

Rancière, Jacques: „Critical Questions on the Theory of Recognition“, in: Deranty, JeanPhilippe / Genel, Katia (Hg.): Recognition or Disagreement. A critical Encounter on the Politics of Freedom, Equality, and Identity, New York 2016, S. 83-95.

Rancière, Jacques: Das Unvernehmen. Politik und Philosophie, Frankfurt/M. 2002.

Rancière, Jacques: Der unwissende Lehrmeister. Fünf Lektionen über die intellektuelle Emanzipation, 2. überarbeitete Auflage, Wien 2009.

Rancière, Jacques: Die Aufteilung des Sinnlichen, 2. Aufl., Berlin 2008.

Rancière, Jacques: La Mésentente. Politique et Philosophie, Paris 1995.

Rancière, Jacques: „Wer ist das Subjekt der Menschenrechte?“, in: Christoph Menke, Francesca Raimondi (Hg.): Die Revolution der Menschenrechte. Grundlegende Texte zu einem neuen Begriff des Politischen, Berlin 2011, S. 474-490.

Stekeler-Weithofer, Pirmin: Philosophie des Selbstbewußtseins. Hegels System als Formanalyse von Wissen und Autonomie, Frankfurt/M. 2005. 
Andreas Gelhard, University of Bonn, Zentrum für Lehrerbildung, Poppelsdorfer Allee 15, D - 53115 Bonn, Germany, e-mail: gelhard@uni-bonn.de

Citation: Gelhard, Andreas: „Bewährung der Gleichheit. Dialektik und radikale Demokratietheorie“, in: Appel, Kurt / Raschke, Carl (eds.): The Crisis of Representation (J-RaT 2018 / 2) pp. 190-211.

Datum der Publikation: 21.01.2019 